\title{
Decision-Dependent Uncertainty in Adaptive Real-Options Water Resource Planning
}

DOI:

10.1016/j.advwatres.2019.103490

\section{Document Version}

Accepted author manuscript

Link to publication record in Manchester Research Explorer

\section{Citation for published version (APA):}

Erfani, T., Pachos, K., \& Harou, J. J. (2020). Decision-Dependent Uncertainty in Adaptive Real-Options Water Resource Planning. Advances in Water Resources, 103490. https://doi.org/10.1016/j.advwatres.2019.103490

\section{Published in:}

Advances in Water Resources

\section{Citing this paper}

Please note that where the full-text provided on Manchester Research Explorer is the Author Accepted Manuscript or Proof version this may differ from the final Published version. If citing, it is advised that you check and use the publisher's definitive version.

\section{General rights}

Copyright and moral rights for the publications made accessible in the Research Explorer are retained by the authors and/or other copyright owners and it is a condition of accessing publications that users recognise and abide by the legal requirements associated with these rights.

\section{Takedown policy}

If you believe that this document breaches copyright please refer to the University of Manchester's Takedown Procedures [http://man.ac.uk/04Y6Bo] or contact uml.scholarlycommunications@manchester.ac.uk providing relevant details, so we can investigate your claim.

\section{OPEN ACCESS}




\title{
Decision-Dependent Uncertainty in Adaptive Real-Options Water Resource Planning
}

\author{
Tohid Erfani ${ }^{\mathrm{a}}$, Kevis Pachos $^{\mathrm{a}}$, Julien J. Harou ${ }^{\mathrm{b}, \mathrm{a}}$ \\ ${ }^{a}$ Department of Civil, Environmental and Geomatic Engineering, University College \\ London, London, UK \\ ${ }^{b}$ School of Mechanical, Aerospace and Civil Engineering, University of Manchester, \\ Manchester, UK
}

\begin{abstract}
Staged water infrastructure capacity expansion optimization models help create flexible plans under uncertainty. In these models exogenous uncertainty can be incorporated into the optimization using an a priori hydrological and demand scenario ensemble. However some water supply intervention uncertainties cannot be considered in this way, such as demand management or technological options. In these cases the uncertainty is endogenous or 'decision-dependent', i.e., the optimized timing and selection of interventions determines when and which uncertainties must be considered. We formulate a multistage real-options water supply capacity expansion optimization model incorporating such uncertainty and describe its effect on cost and option selection.
\end{abstract}

Keywords: Endogenous uncertainty, Adaptive water resources planning

\section{Introduction}

Water security can be threatened when demand increases and climate change reduces supplies. In this case interventions (new infrastructure and/or policies) must be made to meet future demands despite the timing and extent of supply-demand changes not being known in advance. Furthermore, water infrastructures often have long lead-times, such as a decade or more. Traditionally water utilities plan system expansion on a cyclical basis (e.g. every 5 years) aiming to guarantee the supply-demand balance throughout their operating area over a long-term planning period (e.g. 25 years). Generally, given the potential large economic costs of water infrastructure, and the 
uncertainties in both future supplies and demands, formal planning under uncertainty techniques aiming for robustness and/or adaptability are warranted.

Capacity expansion studies are at the heart of water resources engineering (Hsu et al., 2008; Watkins Jr and McKinney, 1998; Guo et al., 2010). In the past a typical water utility expansion plan was a cost-effective schedule of supply- and demand-side capacity expansion actions over the planning horizon (e.g. Padula et al., 2013). The decision-making under uncertainty literature has shifted the goal of water supply planning towards identifying plans that either perform well under a wide range of plausible future conditions (via robust decision making (Lempert, 2003; Lempert et al., 2006; Matrosov et al., 2013b,a)) or are adaptive (i.e., adjusted progressively as new information becomes available (Dupačová, 1995; Ray et al., 2011; Erfani et al., 2018; Hui et al., 2018)). While in the first approach the investment decisions are insensitive to the source of uncertainty, in the latter case, they are optimally activated, delayed and/or replaced so as to meet the supply and demand gap. Approaches that are both robust and adaptive can also be found in the literature (Lempert and Groves, 2010; Haasnoot et al., 2013; Kwakkel et al., 2015).

Most of the optimized water planning under uncertainty literature deals with problems where optimization decisions are independent of the uncertain parameter. That is, the uncertainty is exogenous; e.g. climate change impact that is independent of decisions and is not affected by them., Exogenous uncertainties are usually incorporated as a priori into the multistage optimization problem via an ensemble of scenarios. The earlier work of the authors in Erfani et al. (2018); Pachos et al. (2019) as well as Hall et al. (2012); Mortazavi-Naeini et al. (2014); Borgomeo et al. (2016); Padula et al. (2013); Matrosov et al. (2013b, 2015) are examples of exogenous uncertainty implementation.

Starting from the seminal work of Pflug (1990) and extended later on by the work of Jonsbråten et al. (1998), uncertainty can also be endogenous, meaning that decisions and uncertain parameters are interlinked, or otherwise said, that some uncertainties are decision-dependent, propagating as decisions are made. Based on the work of Pflug (1990); Goel and Grossmann (2006), endogenous uncertainty is of two types; these are described below. 


\subsection{Decision-Dependent uncertainty types}

In dynamic optimization problems where decisions are optimized over time, such as the classical capacity expansion problem, there are two types of decision-dependent uncertainty (also known as 'endogenous uncertainty').

In the first type, intervention options' activation decision variables and the statistical distribution from which the uncertain parameters are derived are dependent. That is, the value of the decision variables cause the alteration of the statistical distribution. This is relevant in water resource management for example for addressing reservoir effects, i.e., when increasing water supply leads to higher water demands which eventually reduce the reservoir's initial water supply improvement (Di Baldassarre et al., 2018). Another example is the application of socio-hydrological models exploring the interplay between the impact of human interventions on drought and flood events and human responses to hydrological extremes (Di Baldassarre et al., 2015, 2017).

In the second type, intervention option activation decisions expressed as binary variables determine when the uncertainty has to be considered (i.e., the binary variables equal one at activation at which point the uncertainty is considered via pre-sampled scenarios). Notable work in this area includes Goel and Grossmann (2004) on oil field development, Viswanath et al. (2004) on network traversal problem, process planning application by Lappas and Gounaris (2016), disaster management by Poss (2014), Nohadani and Sharma (2018), and Peeta et al. (2010), and finally clinical trials modeling by Colvin and Maravelias (2008).

In this paper we modify the adaptive 'real options' water infrastructure planning formulation described by Erfani et al. (2018) to include endogenous uncertainty of the second type where intervention options' activation time determine when their uncertainty must be considered. From now on in this paper, all mentions of 'endogenous uncertainty' refer to this endogenous uncertainty of the second type.

\section{Problem description and formulation}

Figure 1 shows examples of scenario tree structures for a single problem with two options $\mathrm{O}_{1}$ and $\mathrm{O}_{2}$. As can be seen, uncertainty implied by the conditions of $O_{1}$ and $O_{2}$ propagates as and when the activation decisions are made resulting in different scenario tree structures.

To model this problem, we proceed as follows. Let the planning time horizon be a set of discrete time period $t$. Set $I$ covers the sources of endoge- 


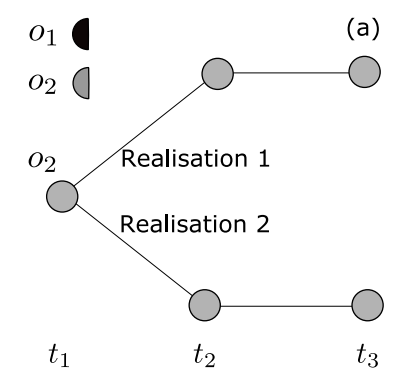

(b)

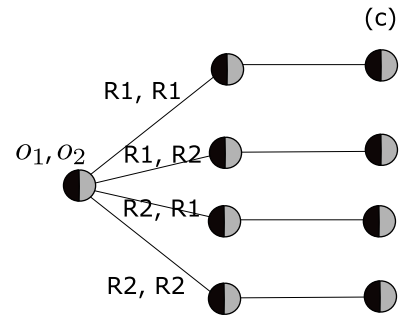

Figure 1: Uncertainty realization for two water development options as endogenous uncertain parameters. In (a) $\mathrm{O}_{2}$ is activated in $t_{1}$ with uncertainty over two possible realizations while $O_{1}$ is never activated accounting for two scenarios. In (b) $O_{1}$ is activated in $t_{1}$ and $\mathrm{O}_{2}$ is activated in $t_{2}$ both with two possible realizations showing three scenarios. In (c) both options are activated in $t_{1}$ and hence produces four scenarios. These activations are during the course of optimization and are not known a priori.

$\min e=\sum_{w \in \Omega, t \in T, i \in I} \frac{p_{w}}{(1+r)^{t}}\left[c C_{i} \times\left(d S_{t, i}^{w}-d S_{t-1, i}^{w}\right)+f C_{i} \times d S_{t, i}^{w}+v C_{i} \times S_{t, i}^{w}\right]$,

s.t.

$$
\begin{aligned}
& \sum_{i \in I} S_{t, i}^{w}+e S_{t}^{w} \geq D_{t}, \quad \forall w \in \Omega, t \in T, \\
& S_{t, i}^{w} \leq d S_{t, i}^{w} \times c S_{i}^{w}, \quad \forall w \in \Omega, t \in T, i \in I, \\
& d S_{t+1, i}^{w} \leq d S_{t, i}^{w}, \quad \forall w \in \Omega, t \in T, i \in I, \\
& d S_{1, i}^{w}=d S_{1, i}^{v}, \quad \forall w, v \in \Omega, i \in I, v \neq w \\
& d S_{t+1, i}^{w}=d S_{t+1, i}^{v} \Leftrightarrow \bigwedge_{i \in D(w, v)} \bigwedge_{l<t}\left(1-d S_{l, i}^{w}\right), \quad \forall w, v \in \Omega, i \in I, v \neq w
\end{aligned}
$$


where $w$ is a scenario with probability of occurrence of $p_{w}, t$ denotes time (stages), $i$ is a water resources development decision, $r$ is the discount rate, $c C_{i}, f C_{i}$ and $v C_{i}$ are respectively the undiscounted capital, fixed, and variable operational costs of investment $i$. The optimization model minimizes the expected cost of intervention options discounted back to the present. Constraint 2 is a mass balance constraint to make sure the sum of existing supplies at time $t, e S_{t}^{w}$ and the supply from water resource option $i$ meets the water demand in time $t, D_{t}$. Constraint 3 allows intervention option $i$ to be used up to its maximum capacity $\left(c S_{i}^{w}\right)$. Constraint 4 forces an irreversible action once activated to remain active until the end of the planning horizon.

Constraint 5 and 6 introduce the endogenous uncertainty. They represent the non-anticipativity constraints (NAC) enforcing that the decisions at time $t$ only utilize any information that is available up to that stage. They do so by linking distinguishable and indistinguishable scenarios. Two scenarios are indistinguishable if they are identical for all uncertain parameters' value that have been manifested up until time $t$. A NAC requires that for those scenarios that are indistinguishable at time $t$, their decisions are the same. Constraint 5 ensures that at the beginning of the first time period $t_{1}$ when no realization of uncertainty has occurred, all scenarios are indistinguishable. Constraint 6 is related specifically to endogenous uncertainty modeling and its implication is explained next.

\subsection{Conditional non-anticipativity constraints}

Constraint 6 is called the Conditional Non-anticipativity Constraint (cNAC). This set of constraints formulates the relationship between the indistinguishable scenarios and the intervention options' decisions. c-NAC ensure that if scenarios are indistinguishable, then NAC is enforced and if not, they are ignored. To do so we define the set $D$ in constraint 6 for scenario $v$ and $w$ in $\Omega$ as:

$$
D(w, v)=\left\{i \mid i \in I, \theta_{i}^{w} \neq \theta_{i}^{v}\right\} .
$$

$D$ represents a set of decisions in which scenario $w$ and $v$ differ in their possible realization. Under constraint 6 , if there is no activation decision in those $i$ that distinguish scenario $w$ and $v$ by time $t, w$ and $v$ are marked indistinguishable using $d S_{i, t}^{*}$.

Due to constraint 6 , the proposed formulation is a logical disjunctive programming model. The logical constraint is due to the conditionality of 
the NAC, and the disjunctive constraint is because of the distinguishability of scenarios. Such a model can be reformulated to mixed integer programming using the convex hull reformulation described in Williams (2013).

\section{Application to a water resource planning problem}

To illustrate an application of the above formulation we consider a water company with three investment decisions to implement with a five time-step planning horizon. We consider the case in which the demand growth and existing supply projection are known (Table 1). However, the intervention options include both demand management and supply expansion options (Table 2 ). The extra capacity added to the system is achieved via demand management (decreasing the water demand) and supply expansion options. The formulation is a least-cost aggregate supply-demand, as per Erfani et al. (2018). The uncertainties implied by the water supply-demand intervention options follow a triangular distribution. We use three realizations and mark each level as low, medium and high shown in Table 2. The distribution reflects all the possible scenarios of future realization of water availability at the time an intervention is selected. In practice such distributions of how much water a source can supply are estimated via joint hydrological and water resource systems modeling (Padula et al., 2013).

Table 1: Existing water availability and demand growth projection

\begin{tabular}{rccccc}
\hline & $t_{1}$ & $t_{2}$ & $t_{3}$ & $t_{4}$ & $t_{5}$ \\
\hline Demand $(\mathrm{Ml} / \mathrm{d})$ & 2010 & 2024 & 2042 & 2050 & 2060 \\
Water availability $(\mathrm{Ml} / \mathrm{d})$ & 2000 & 2000 & 2000 & 2000 & 2000 \\
\hline
\end{tabular}

Table 2: Decision dependent uncertainty implied by the investment options

\begin{tabular}{ccccc}
\hline \multicolumn{5}{c}{ Water availability by expanding capacity $(\mathrm{Ml} / \mathrm{d})$} \\
\hline Intervention & high & medium & low & Mean \\
\hline$O_{1}$ & 60 & 42 & 40 & 47 \\
$O_{2}$ & 25 & 20 & 5 & 17 \\
$O_{3}$ & 20 & 18 & 15 & 18 \\
\hline
\end{tabular}




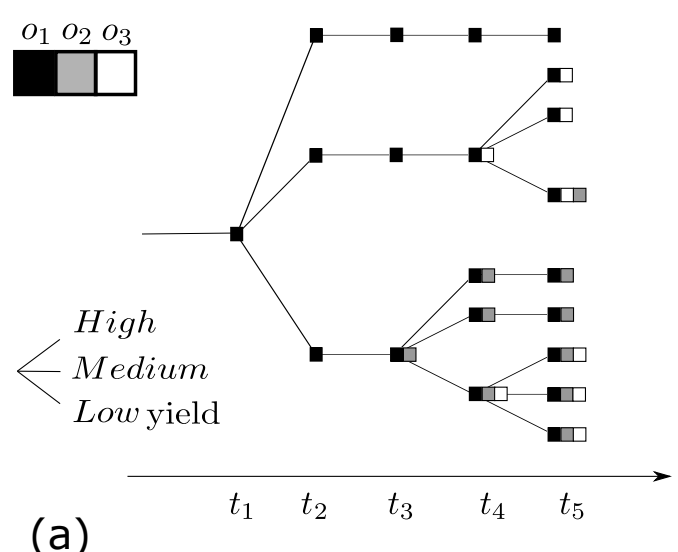

(a)

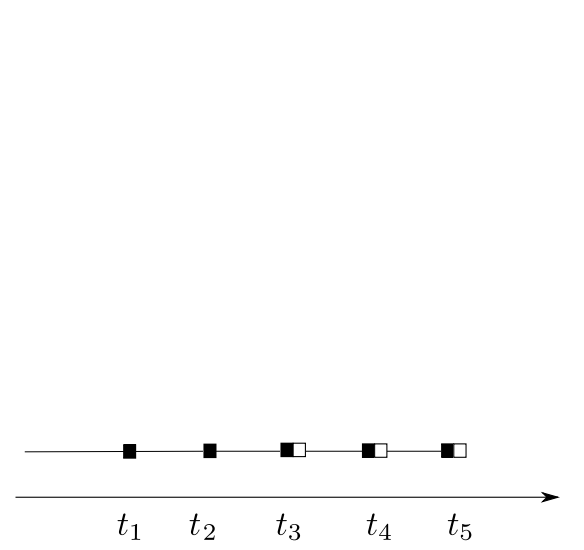

(c)

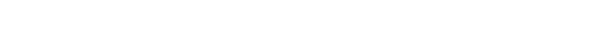

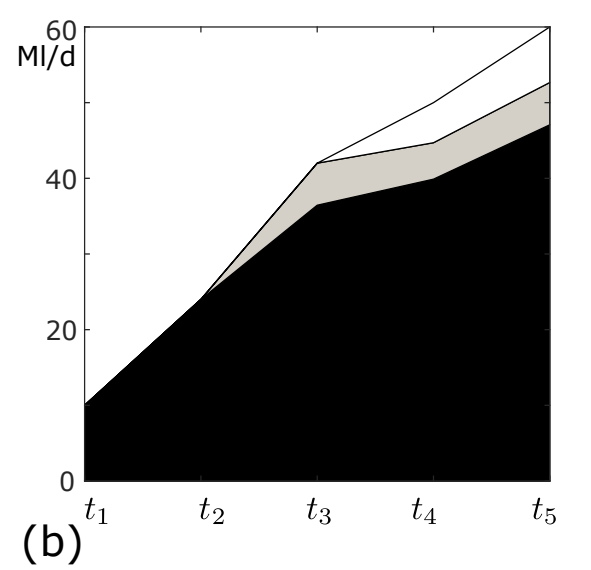

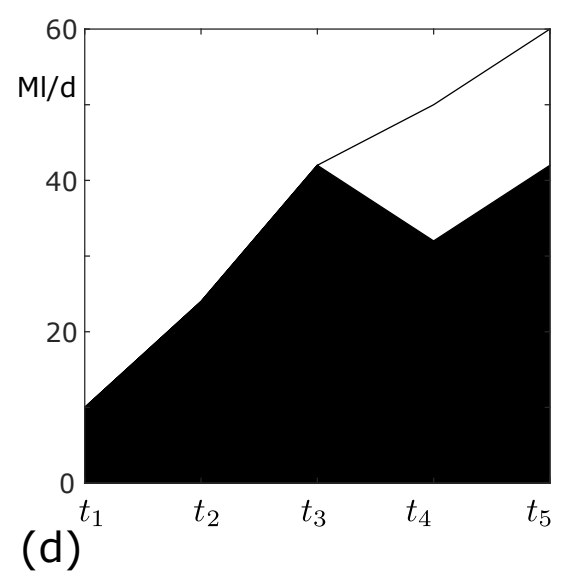

Figure 2: (a) Solution structure for capacity expansion by considering endogenous uncertainty, (b) Utilization of options by considering endogenous uncertainty, (c) Capacity expansion deterministic solution, and (d) Utilization of options activated in deterministic solution 


\section{Results and discussion}

The optimal activation of options and their utilization are shown in Figure 2. We compare the solutions of the proposed model with those of the deterministic one where the mean value of the uncertain parameter is used for all development options. The deterministic solution (shown in Figure 2.c) suggests investing in option 1 at the beginning of the planning period and to supplement the portfolio with $o_{3}$ from time period 3 onwards. In contrast to the deterministic solution in which $o_{3}$ is always activated in time period 4 and 5 , commitment to $o_{3}$ is only required as an optimal recourse decision in the endogenous uncertainty model (Shown in Figure 2.a) if either $o_{1}$ and $o_{2}$ are realized at low level or $o_{1}$ is at its medium level. In addition, compared to the deterministic solution in which option activation in $O_{2}$ is never suggested, in the endogenous uncertainty model, investment in $O_{2}$ is either delayed to the last stage, if $o_{1}$ and $o_{3}$ are realized at medium and low level, respectively, or, $o_{1}$ is at its low level. This flexibility in options' activation is valuable because by not selecting an investment option now and deferring it to the next planning period, asset managers avoid its cost until more information is available. Indeed, the expected cost of the proposed formulation is $10 \%$ lower than the deterministic one suggesting the economic value of flexibility in our case study. This highlights the value of including endogenous uncertainty, and how much it is worth to postpone a decision until more information is available. By not committing to $o_{3}$ in time period 3, planners can postpone investment until later, when and if it is required. For the application of the proposed method to a real case study it could be useful to explore the sensitivity of optimal pathways to the selection of the probability distributions, which cannot be assumed to be exact.

\section{Extended formulation}

In order to simplify the explanation of endogenous uncertainty our synthetic case-study assumed no exogenous uncertainties such as the one described by Erfani et al. (2018). That is, in the illustrious example provided here projections of existing supply capacity and demand growth are deterministic. To make this approach applicable for a problem with both types of uncertainties, for any individual realization of exogenous uncertain parameter (through the scenarios), all possible realizations of endogenous parameters should be included. To formalize this, assume that $\xi_{t}$ is the vector of exogenous uncertain parameters associated with time period $t$. $\Xi$ is discrete 
set of possible realizations for vector $\xi=\left(\xi_{1}, \ldots, \xi_{T}\right)$ represents the set of all exogenous uncertainty scenarios. The scenario in a problem formulation with both exogenous and endogenous uncertainty elements corresponds now to one possible realization for vector $\left(\xi_{1}, \ldots, \xi_{T}, \theta_{1}, \ldots, \theta_{I}\right)$. With this amendment, $\Omega$ is now a set of all the endogenous and exogenous scenarios given by the Cartesian product of both exogenous and endogenous scenario sets $\Omega$ $\left.=\left(\times_{i \in I}\right) \Theta_{i}\right) \times \Xi$; i.e., for any realization of the vector of exogenous paratemers $\xi$, the set of scenarios includes scenarios corresponding to all possible combinations of realizations for the endogenous parameters. $\theta_{i}^{w}$ and $\xi_{t}^{w}$ will represent the realizations of $\theta_{i}$ and $\xi_{t}$, respectively, in scenario $w$. Note that $\theta_{i}$ is not time (but decision) dependant while $\xi_{t}$ is independent of decisions and is resolved on given time $t$. We add the following set of equations to problem of section 2 to include both exogenous and endogenous uncertainty:

$$
d S_{t+1, i}^{w}=d S_{t+1, i}^{v}, \quad \forall w, v \in \Xi, t \in T, i \in I, v \neq w
$$

where constraint 8 is the NAC for exogenous uncertainty. If we do not have endogenous uncertainty, then $\Theta$ is an empty set and the above problem reduces to exogenous model (as explained in Erfani et al. (2018)). Similarly, if there is no exogenous parameters, then we have $\Xi=\emptyset, \Omega=\Theta$, and model reduces to the endogenous model (as explained by the model in Section 2). Adding both uncertainties would increase the size of the problem mainly due to the fact that the non-anticipativity constraints, which account for most constraints, grow quadratically with the number of scenarios. The size of the problem could be reduced using different theoretical approaches including the property of the set $D$, referring to the work of Gupta and Grossmann (2011), where an asymmetric structure of matrix $D$ proves many NACs redundant.

\section{Conclusion}

This paper proposed an extension to an adaptive multistage real options water infrastructure planning optimization problem formulation for when some uncertainties are endogenous. That is, problems where water resource system intervention decisions control when additional uncertainties associated with new options must be introduced. The proposed formulation is demonstrated on a synthetic problem with a small number of options showing how endogenous uncertainty propagates when making planning decisions 
over time. The results are compared with the deterministic formulation in terms of option activations and the expected present value of the cost; the formulation with endogenous uncertainty saves 10\%. For simplicity in presenting the endogenous uncertainty concept, the case-study assumed no exogenous uncertainties and referred the challenge of applying the extended formulation to cases with both exogenous and endogenous uncertainties to future work. This includes dealing with a larger multistage optimization problem as well as the correlation between uncertain parameters.

\section{References}

\section{References}

Borgomeo, E., Mortazavi-Naeini, M., Hall, J.W., O’Sullivan, M.J., Watson, T., 2016. Trading-off tolerable risk with climate change adaptation costs in water supply systems. Water Resources Research .

Colvin, M., Maravelias, C.T., 2008. A stochastic programming approach for clinical trial planning in new drug development. Computers \& Chemical Engineering 32, 2626-2642.

Di Baldassarre, G., Martinez, F., Kalantari, Z., Viglione, A., 2017. Drought and flood in the anthropocene: feedback mechanisms in reservoir operation. Earth System Dynamics 8, 1-9.

Di Baldassarre, G., Viglione, A., Carr, G., Kuil, L., Yan, K., Brandimarte, L., Blöschl, G., 2015. Debates-perspectives on socio-hydrology: Capturing feedbacks between physical and social processes. Water Resources Research $51,4770-4781$.

Di Baldassarre, G., Wanders, N., AghaKouchak, A., Kuil, L., Rangecroft, S., Veldkamp, T.I., Garcia, M., van Oel, P.R., Breinl, K., Van Loon, A.F., 2018. Water shortages worsened by reservoir effects. Nature Sustainability 1,617 .

Dupačová, J., 1995. Multistage stochastic programs: The state-of-the-art and selected bibliography. Kybernetika 31, 151-174. 
Erfani, T., Pachos, K., Harou, J., 2018. Real-options water supply planning: Multistage scenario trees for adaptive and flexible capacity expansion under probabilistic climate change uncertainty. Water Resources Research

Goel, V., Grossmann, I.E., 2004. A stochastic programming approach to planning of offshore gas field developments under uncertainty in reserves. Computers \& chemical engineering 28, 1409-1429.

Goel, V., Grossmann, I.E., 2006. A class of stochastic programs with decision dependent uncertainty. Mathematical programming 108, 355-394.

Guo, P., Huang, G., Li, Y., 2010. An inexact fuzzy-chance-constrained twostage mixed-integer linear programming approach for flood diversion planning under multiple uncertainties. Advances in Water Resources 33, 81-91.

Gupta, V., Grossmann, I.E., 2011. Solution strategies for multistage stochastic programming with endogenous uncertainties. Computers \& Chemical Engineering 35, 2235-2247.

Haasnoot, M., Kwakkel, J.H., Walker, W.E., ter Maat, J., 2013. Dynamic adaptive policy pathways: A method for crafting robust decisions for a deeply uncertain world. Global environmental change 23, 485-498.

Hall, J.W., Lempert, R.J., Keller, K., Hackbarth, A., Mijere, C., McInerney, D.J., 2012. Robust climate policies under uncertainty: A comparison of robust decision making and info-gap methods. Risk Analysis 32, 16571672 .

Hsu, N.S., Cheng, W.C., Cheng, W.M., Wei, C.C., Yeh, W.W.G., 2008. Optimization and capacity expansion of a water distribution system. Advances in Water Resources 31, 776-786.

Hui, R., Herman, J., Lund, J., Madani, K., 2018. Adaptive water infrastructure planning for nonstationary hydrology. Advances in water resources $118,83-94$.

Jonsbråten, T.W., Wets, R.J., Woodruff, D.L., 1998. A class of stochastic programs withdecision dependent random elements. Annals of Operations Research 82, 83-106. 
Kwakkel, J.H., Haasnoot, M., Walker, W.E., 2015. Developing dynamic adaptive policy pathways: a computer-assisted approach for developing adaptive strategies for a deeply uncertain world. Climatic Change 132, 373-386.

Lappas, N.H., Gounaris, C.E., 2016. Multi-stage adjustable robust optimization for process scheduling under uncertainty. AIChE Journal 62, 1646-1667.

Lempert, R.J., 2003. Shaping the next one hundred years: new methods for quantitative, long-term policy analysis. Rand Corporation.

Lempert, R.J., Groves, D.G., 2010. Identifying and evaluating robust adaptive policy responses to climate change for water management agencies in the american west. Technological Forecasting and Social Change 77, 960-974.

Lempert, R.J., Groves, D.G., Popper, S.W., Bankes, S.C., 2006. A general, analytic method for generating robust strategies and narrative scenarios. Management science 52, 514-528.

Matrosov, E.S., Huskova, I., Kasprzyk, J.R., Harou, J.J., Lambert, C., Reed, P.M., 2015. Many-objective optimization and visual analytics reveal key trade-offs for london's water supply. Journal of Hydrology 531, 1040-1053.

Matrosov, E.S., Padula, S., Harou, J.J., 2013a. Selecting portfolios of water supply and demand management strategies under uncertainty - contrasting economic optimisation and 'robust decision making'approaches. Water resources management 27, 1123-1148.

Matrosov, E.S., Woods, A.M., Harou, J.J., 2013b. Robust decision making and info-gap decision theory for water resource system planning. Journal of Hydrology 494, 43-58.

Mortazavi-Naeini, M., Kuczera, G., Cui, L., 2014. Application of multiobjective optimization to scheduling capacity expansion of urban water resource systems. Water Resources Research 50, 4624-4642.

Nohadani, O., Sharma, K., 2018. Optimization under decision-dependent uncertainty. SIAM Journal on Optimization 28, 1773-1795. 
Pachos, K., Erfani, T., Huskova, I., Matrosov, E., Harou, J., 2019. Accommodating flexibility and adaptability in multi-objective optimised water supply planning. Water Resources Research (under review) .

Padula, S., Harou, J.J., Papageorgiou, L.G., Ji, Y., Ahmad, M., Hepworth, N., 2013. Least economic cost regional water supply planning-optimising infrastructure investments and demand management for south east england's 17.6 million people. Water resources management 27, 5017-5044.

Peeta, S., Salman, F.S., Gunnec, D., Viswanath, K., 2010. Pre-disaster investment decisions for strengthening a highway network. Computers \& Operations Research 37, 1708-1719.

Pflug, G.C., 1990. On-line optimization of simulated markovian processes. Mathematics of Operations Research 15, 381-395.

Poss, M., 2014. Robust combinatorial optimization with variable cost uncertainty. European Journal of Operational Research 237, 836-845.

Ray, P., Kirshen, P., Watkins Jr, D., 2011. Staged climate change adaptation planning for water supply in amman, jordan. Journal of Water Resources Planning and Management 138, 403-411.

Viswanath, K., Peeta, S., Salman, S.F., et al., 2004. Investing in the links of a stochastic network to minimize expected shortest path. length. Technical Report. Purdue University, Department of Economics.

Watkins Jr, D.W., McKinney, D.C., 1998. Decomposition methods for water resources optimization models with fixed costs. Advances in Water Resources 21, 283-295.

Williams, H.P., 2013. Model building in mathematical programming. John Wiley \& Sons. 